\title{
Comparison of original gram stain and its modification in the gingival plaque samples
}

\begin{abstract}
Aim: The aim of the study is to compare the characteristics of original gram stain and its modifications in the gingival plaque samples.

Background: The gram stain remains the most frequently used rapid diagnostic test. It is described as the corner stone of clinical laboratory. The gram stain divides bacteria into gram positive and gram negative on the basis of their cell wall and cell membrane permeability. It is a very important preliminary step in the initial characterization and classification of bacteria. When properly interpreted in the light of clinical history, the gram stain can provide useful, presumptive information as to the etiology of much infection. Various modifications have been made in the staining pattern for better diagnosis of the micro organisms.
\end{abstract}

Materials and methods: Seven paired gingival plaque samples are collected from seven healthy individuals. One set labeled as original gram and the other set labeled as modified gram. Both the set are heat fixed and stain with two different methods and scored by two different observers who are blind to the study.

Results and conclusion: $80.95 \%$ of the smear shows good Staining intensity in modified gram stain as compared to the original gram because acetone alcohol mixture gives a better decolourising agent than the normal acetone. $89.27 \%$ of the smear demonstrates organism well with more than 50 bacteria per smear. Modification of the original gram stain gives a better staining intensity and demonstrates the organism well which can help in better diagnosis.

Keywords: gram stain, modification, staining intensity, diagnosis
Volume 7 Issue I - 2019

Nirmada pukhrambam

Department of Oral Pathology, Saveetha Dental College, India

Correspondence: Nirmada pukhrambam, Department of Oral Pathology, Saveetha Dental College, India, Email nirmadapukhrambam@gmail.com

Received: October 03, 2018 | Published: January 22, 2019

\section{Introduction}

The gram stain is one of the most frequently used diagnostic tests. It was first described by Danish pathologist Christian Gram in 1884. It is described as the corner stone of clinical laboratory. ${ }^{1}$ the gram stain divides bacteria into gram positive and gram negative on the basis of their cell wall and cell membrane permeability.

The principle of gram stain is that some organisms are not decolorized and retain color of basic stain; example gentian violet (gram positive organisms) while the others lose all gentian violet and take up the counter stain; example dilute carbol fuschion or safranin (gram negative organisms).

The bacterial cell envelope is a complex multi layered structure that serves to protect these organisms from their unpredictable and often hostile environment. Bacterial cell structure: surface layerscell wall, cell membrane, capsule, appendages-flagella, pili or fimbri cytoplasm- nuclear material, ribosomes, embosomed, inclusions and special structure-endospore.

The gram positive cell wall has a thick mesh like cell wall made up of peptidoglycan. Peptidoglycan is a polysaccharide composed of two sub units called N-acetyl glucosamine and $\mathrm{N}$-acetyl muramic acid. Peptidoglycan layers are cross linked by short chains of peptides by means of a transpeptidase enzyme, which makes the cell wall rigid. Lipoteichoic acid is another constituent of the cell wall that is present between the peptidoglycan layers. Lipoteichoic acids act as a regulator of autolytic cell enzymes. It has also antigenic properties that stimulate specific immune responses when it is released from the cell wall after cell death.
Gram negative bacteria have a thinner layer of peptidoglycan $(10 \%$ of the cell wall). They have an outer membrane which contains lipids which is separated from the cell wall by periplasmic space. ${ }^{1}$ Gram stain interpretation gives information about the presence or absence of bacterial disease and can guide the initial antibiotic treatment. Gram stain also provides additional information about the host immune response and quality of the specimen. A well prepared sample can show the organism colour size, shape and arrangement, allowing cellular morphology to further separate bacteria into four major groups. Cocci are spherical or oval, bacilli are rod like or cylindrical, vibrios are comma shaped or curved like and spirochetes are flexible.

The original gram stain also has certain limitations. The original gram stain has certain limitations in identification of some organisms. Sometimes gram positive organisms may appear gram negative or vice versa due to excessive heat, prolonged decolourisation etc. and one more limitation is, it cannot be applied in tissue sections.

Many modifications of the original gram staining technique have been published. Some of them have improved the method, while others include some minor technical variants of no value. The test developed by Christain Gram in 1884 was modified by Hucker in 1921. The modified procedure provides greater reagent stability and better differentiation of organisms. ${ }^{2}$

Hence the study aims to compare the characteristics of original gram stain and its modifications in terms of staining intensity, organism demonstration, and background staining and cells morphology in the gingival plaque samples. 


\section{Materials and methods}

Seven paired gingival plaque samples were collected from seven healthy individuals. All the smears were heat fixed and stain by the following procedures.

\section{Original gram stain:}
i. Methyls violet 1 min- washes in.
ii. Iodine- $1 \mathrm{~min}-$ wash.
iii. Acetone add drop by drop -wash
iv. Dilute carbol fuschion-1min-wash.
v. Allow to dry.
vi. Examine under (100x).

\section{Modified gram stain:}
i. Crystal violet-30sec.
ii. Iodine 30 sec-wash.
iii. Acetone- alcohol 1-5sec-wash immediately.
iv. Safranin-30sec- wash
v. Allow to dry.
vi. Examine under 100xs.

Both the smears are scored by two different observers who are blind to the study.

\section{Number of bacteria:}
i. Grade1-<bacteria.
ii. Grade2- 2 to 10 bacteria.
iii. Grade3 - 11 to 50 bacteria.
iv. Grade4- $>50$ bacteria.

\section{Demonstration of cells:}
i. Grade1- $<1$ cells.
ii. Grade2- 1 to 5 cells.
iii. Grade3- 6 to 10 cells.
iv. Grade4 - >10 cells.

\section{Staining intensity:}
i. Grade 1- poor.
ii. Grade2- acceptable.
iii. Grade 3-good.

\section{Results}

Comparing the overall four characteristic of staining, modified gram stain was found to have better results than the original gram stain. The two observer scores using kappa statistics showed $78.05 \%$ that is good agreement is established between the observers.

\section{Statistical analysis}

Data collected were tabulated and statistically analyzed using interobserver variability test (kappa statistics). The graphical representation is given in Figure 1-3. Table 1 shows the interobserver agreement between the two observers.

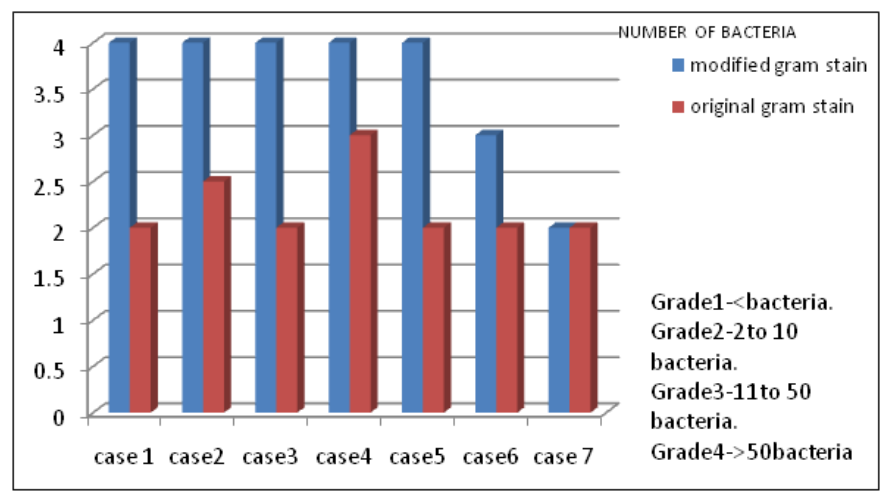

Figure I Demonstration of number of bacteria.

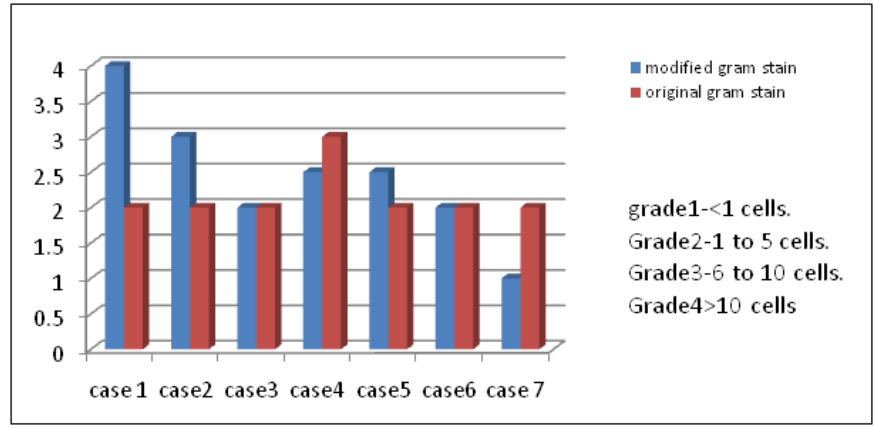

Figure 2 Demonstration of cells.

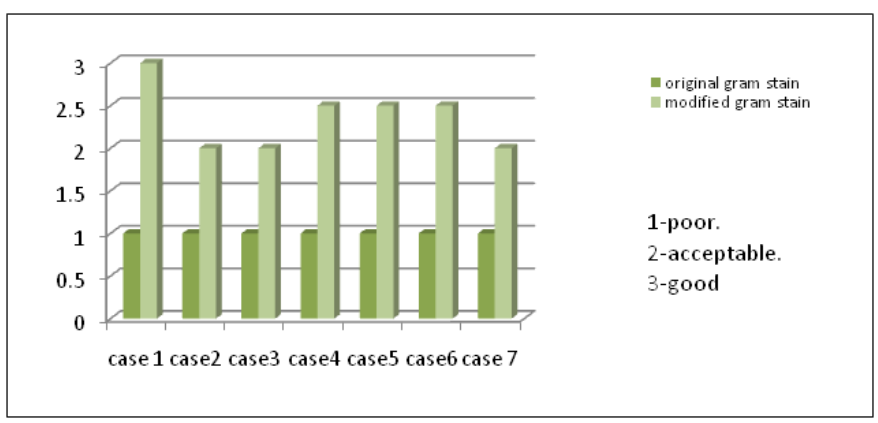

Figure 3 Staining intensity.

Table I Interobserver agreement between the two observers.

\begin{tabular}{ll}
\hline Characters & Interobserver agreement (kappa) \\
\hline Staining intensity & 0.222 \\
Demonstration of organism & 1.000 \\
Demonstration of cells. & 0.000 \\
\hline
\end{tabular}

\section{Discussion}

In modified gram stain, we used crystal violet as the primary stain and decolouriser as acetone alcohol mixture with reduction in the staining time. $80.95 \%$ of the smear shows good Staining intensity in modified gram stain as compared to the original gram because acetone alcohol mixture gives a better decolourising agent than the normal acetone as it dissolves the lipids found in the outer cell layer of gram negative bacteria allowing the crystal violet to leak out.

$89.27 \%$ of the smear demonstrates organism well with more than 50 bacteria per smear. This is because the background staining 
intensity is highly improved in the modified stain by reduction in the counter staining time to 30 seconds. $60.71 \%$ of the smears in modified stain shows more than 10 cells per field as compared to original gram which is observed in only $43.5 \%$ of the smears.

$80.95 \%$ of the smear shows good Staining intensity in modified gram stain as compared to the original gram because acetone alcohol mixture gives a better decolourising agent than the normal acetone as it dissolves the lipids found in the outer cell layer of gram negative bacteria allowing the crystal violet to leak out.

$60.71 \%$ of the smears in modified stain shows more than 10 cells per field as compared to original gram which is observed in only about $43.5 \%$ of the smears. No study about the modification in original gram stain has been reported in literature so far. Further study with more increase in sample size is needed to prove the effectiveness of the modified method.

\section{Conclusion}

Modification of the original gram stain gives a better staining intensity and demonstrates the organism well which can help in better diagnosis. ${ }^{3}$

\section{Acknowledgment}

None.

\section{Conflicts of interest}

There is no conflict of interest among the authors.

\section{References}

1. Thairu Y, Nasir IA, Usman Y. laboratory perspective of gram staining and its significance in investigations of infectious diseases. Afr $\mathrm{J} \mathrm{Med}$. 2014;1(4):168-174.

2. Michael PD. Disorders of Glucose Metabolism in Patients Infected with Human Immunodeficiency Virus. Clinical infectious diseases. 2000;31(6):1467-1475.

3. Rita BM, Jackie R, Donald PB. Differential Staining of Bacteria: Gram Stain. Current Protocols in microbiology. 2009;15(1):3-8. 